\title{
Oleaginous yeasts form Uruguay and Antarctica as renewable raw material for biodiesel production
}

\author{
Virginia Pereyra ${ }^{1}$, Adalgisa Martinez ${ }^{1}$, Caterina Rufo ${ }^{2}$, Silvana Vero, ${ }^{1, *}$ \\ ${ }^{1}$ Catedra de Microbiología, Departamento de Biociencias, Facultad de Química, Universidad de la República, Gral Flores 2124, Montevideo, \\ Uruguay \\ ${ }^{2}$ Instituto Polo Tecnológico, Facultad de Química, Universidad de la República, By Pass Ruta 8 s/n, Pando, Canelones, Uruguay
}

Email address:

vipereyra@gmail.com (V. Pereyra), adalgisamar@gmail.com (A. Martínez), crufo@fq.edu.uy (C. Rufo), svero@fq.edu.uy (S. Vero)

\section{To cite this article:}

Virginia Pereyra, Adalgisa Martinez, Caterina Rufo, Silvana Vero. Oleaginous Yeasts form Uruguay and Antarctica as Renewable Raw Material for Biodiesel Production. American Journal of BioScience. Vol. 2, No. 6, 2014, pp. 251-257. doi: 10.11648/j.ajbio.20140206.20

\begin{abstract}
In the present study a screening of oleaginous yeasts from different habitats nearby Montevideo, Uruguay, was carried out. Four yeast strains previously isolated from soil from Fildes Peninsula, in the Antarctic region, were also included in the study. More than $75 \%$ of the obtained isolates were characterized as oleaginous though only four of them (two from Antarctica and two from Uruguay) were able to accumulate lipids to levels exceeding the $40 \%$ of cell dry weight. One of the selected strains was identified as Rhodotorula graminis, which has been already recognized as an oleaginous species. However, the other three isolates belong to species, which have not been reported as oleaginous before. This work constitutes the first report of Cryptococcus phenolicus, Cystofilobasidium infirmominiatum and Leucosporidium scottii as oleaginous species. Three of the selected isolates were able to grow with glycerol as carbon source. According to lipid production in presence of glycerol, one isolate was selected for further studies. The ability to grow and accumulate intracellular lipids in presence of crude glycerol was assessed for the selected strain. In such conditions, a maximum concentration of $5.9 \mathrm{~g} \mathrm{~L}^{-1}$ of lipids with a suitable fatty acid profile according to the requirements established in our country for the raw material used in the production of biodiesel, was obtained.
\end{abstract}

Keywords: Yeasts, Biodiesel, Glycerol

\section{Introduction}

The depletion of fossil fuel reserves alongside an increase in energy demand calls for a greater supply of fuels from renewable resources. As a result, the production of biofuels like bioethanol or biodiesel has increased worldwide during the last decade. Biodiesel consists of the alkyl esters of fatty acids that are typically produced from a combination of triglycerides (e.g. vegetable oils) and an alcohol (e.g., methanol) in the presence of either a base or acid catalyst. In the production process, glycerol is also formed, representing $10 \%$ of the product output [1]. The dramatic growth of the biodiesel industry has created a surplus of glycerol, which has resulted in an important decrease in crude glycerol prices over the last few years and generated environmental concerns associated with contaminated glycerol disposal [2]. The crude glycerol obtained from biodiesel production is typically contaminated with unreacted methanol, so it must be refined to recover the alcohol. That process leaves a crude glycerol at about $80 \%$, which also contains salts and residues of methanol of about $3 \%$, so further refining is needed to obtain pure glycerol. Purifying it is costly and generally out of the range of economic feasibility for the small to medium sized plants [3]. Therefore, it is very important to find alternative fates to this low-grade glycerol, called crude glycerol to further defray the cost of biodiesel production in the growing global market.

At present, biodiesel is mainly produced from vegetable oils, which are not economically competitive with petroleumbased fuel because of the relatively high cost of the oil feedstock. In fact, the cost of source accounts for 70-85\% of the entire production cost. Moreover, this kind of production competes for agricultural land that can be used instead for food production. Microbial oils produced by "oleaginous" microorganisms, including bacteria, yeasts, molds and algae, are now believed to be a promising potential source for biodiesel production. It was demonstrated that "oleaginous" microorganisms can accumulate intracellular triglycerides (more than $20 \%$ of biomass weight), with a fatty acid 
composition very similar to that of vegetable oils [4]. The culture of oleaginous microorganisms involves fewer costs than oil crop production, is affected neither by seasons nor by climates, and is environmentally friendly since no pesticide applications are needed. Moreover, production cycles are far shorter, involving days instead of months in the case of vegetable crops.

This work aims to select an oleaginous yeast strain that is able to accumulate intracellular saponifiable lipids suitable for biodiesel production when grown with crude glycerol as carbon source.

\section{Materials and Methods}

\subsection{Source of Yeasts}

Yeasts used in this work were isolated from soil samples nearby Montevideo, Uruguay and from the surface of different fruits from local orchards. Ten grams of soil or fruit peel were homogenized in $100 \mathrm{ml}$ of sterile water. Ten microliters of the obtained suspensions were streaked onto Dichloran Bengal Rose chloramphenicol Agar (Difco, Detroit, MI, USA) plates and incubated 6 days at $28{ }^{\circ} \mathrm{C}$. Colonies corresponding to yeasts were streaked onto Potato Dextrose Agar (PDA) (Difco) plates to obtain pure cultures. Five yeast strains (Leucosporidium scottii At17, Cryptococcus gastricus At4, Rhodotorula mucilaginosa At7, Rhodotorula laryngis At16, Cystofilobasidium infirmominiatum PL1) from the Microbiology Department of Facultad de Química culture collection were also included in this study. The four first strains were isolated from Antarctic soil samples[5], while the fifth was isolated from lemon surface and characterized as a good biocontrol agent of blue mold of oranges[5].

\subsection{Screening for Oleaginous Yeasts}

Lipid accumulation capacity of yeast isolates was assessed using the Sudan Black B lipid staining technique [6]. Each yeast strain was grown at $28{ }^{\circ} \mathrm{C}$ in a rotary shaker at $150 \mathrm{rpm}$ in $250 \mathrm{~mL}$ Erlenmeyer flasks containing $50 \mathrm{~mL}$ of a liquid nitrogen-limited medium defined by Thakur et al. [7]. After 5 days of incubation, Sudan Black-stained smears from each culture were observed under optical microscopy looking for the presence of intracellular blue oil globules which are characteristic of oleaginous yeasts. Non oleaginous yeast (Saccharomyces cerevisiae) was used as a negative control [8]. For the oleaginous isolates, intracellular content of saponifiable lipids was quantified as described by Burja et al. [9] with modifications. At the same time, biomass concentration was determined gravimetrically as dry weight and percentage of lipid accumulation in cell biomass was calculated for each isolate. All experiments were repeated twice. Data was analyzed by one-way ANOVA, and mean separations were performed by LSD tests. Differences at $\mathrm{P}<0.05$ were considered significant. Those isolates, which accumulated significantly higher amounts of intracellular lipids, were selected.

\subsection{Determination of Fatty Acid Profiles}

Fatty acids obtained as described above were esterified with methanol under acidic conditions as described by Burja et al.[9]. Fatty acid methyl esters (FAMEs) were analyzed by gas chromatography using a Shimadzu GC 2014 equipped with a Carbowax $20(30 \mathrm{~m}, 0.25 \mathrm{~mm}$ ID, $0.25 \mu \mathrm{m})$ column and a flame ionization detector. Operation conditions were: $\mathrm{N}_{2}$ carrier gas $40 \mathrm{ml} \mathrm{min}{ }^{-1}$, injection port temperature $230{ }^{\circ} \mathrm{C}$, oven temperature $190{ }^{\circ} \mathrm{C}$ and the detector temperature was 230 ${ }^{\circ} \mathrm{C}$. FAMEs identity was determined by comparison of retention times with those obtained with known standards (GLC 10 FAME mix, Sigma-Aldrich 1891). Quantitation was carried out using peak area integrals expressed as a percentage of the total of all integrals. The methyl esters that were not present in the standard mixture were identified by the retention time and the fragmentation pattern data of GC-MS analysis using a QP-2010 Shimadzu, with a column AT-5ms (30 $\mathrm{m} \times$ $0.25 \mathrm{~mm}, 0.10 \mu \mathrm{m})$. Oven temperature was programmed from $150{ }^{\circ} \mathrm{C}$ to $215^{\circ} \mathrm{C}$ at $1.25^{\circ} \mathrm{C} \mathrm{min}{ }^{-1}$, and then to $230{ }^{\circ} \mathrm{C}$ at $25^{\circ} \mathrm{C}$ $\mathrm{min}^{-1}$, the flow rate of carrier gas was set at $31 \mathrm{ml} \mathrm{min}^{-1}$. Other settings were as follows: $300^{\circ} \mathrm{C}$ of interface temperature, 250 ${ }^{\circ} \mathrm{C}$ of injection temperature, and electron impact ionization (EI) at $70 \mathrm{eV}$. Cetane number $(\mathrm{CN})$ of fatty acid methyl esters obtained for each isolate was calculated empirically following the procedure of Azam et al. [10].

\subsection{Identification and Characterization of the Selected Oleaginous Yeast Strains}

Selected oleaginous strains were identified by sequence analysis of the D1/D2 variable domains at the $5^{\prime}$ end of the large subunit rRNA gene (D1/D2) and the internal transcribed spacers, ITS 1 and 2, including the 5.8S rDNA sequence. DNA extraction was carried out as described by Schena et al. (1999) [11]. PCR fragments were generated using primers ITS1[12] and D2R covering both the ITS1ITS2 and D1/D2 regions [13]. The thermal profile was $96^{\circ} \mathrm{C}$ $2 \mathrm{~min}$, followed by 35 cycles of $96{ }^{\circ} \mathrm{C} 30 \mathrm{~s}, 51^{\circ} \mathrm{C} 45 \mathrm{~s}, 72^{\circ} \mathrm{C}$ $120 \mathrm{~s}$, and a final extension step at $72{ }^{\circ} \mathrm{C}$ for $7 \mathrm{~min}$. Nucleotide sequences of the PCR products were determined in both directions at Macrogen (Macrogen Inc., Seoul, Korea). Sequences were aligned with MEGA 5 [14], visually corrected and compared to NCBI databases using BLAST. Phylogenetic analyses of D1/D2 and ITS1-ITS2 sequences of the selected isolates were conducted using MEGA version 5 [14]. DNA sequences were aligned with sequences of homologous regions of closely related strains retrieved from GenBank. Evolutionary distances were computed using the Jukes-Cantor method [15], and phylogenetic trees were obtained by neighbor-joining[16]. All positions containing alignment gaps and missing data were eliminated in pairwise sequence comparisons (pairwise deletion option). Stability of clades was assessed with 1000 bootstrap replications [17].

\subsection{Intracellular Lipid Accumulation in Presence of Glycerol}

The ability to use glycerol as an only carbon source was 
assessed for the selected strains, according to Kurtzman and Fell [18]. Yeast isolates capable of growing in such conditions were selected and cultivated in lipid accumulating conditions as described above [11], but substituting glucose for glycerol. Intracellular content of saponifiable lipids and fatty acid profiles of those lipids were determined at the fifth day of culture, as described above. All experiments were repeated three times. Data corresponding to the amount of fatty acids from saponifiable lipids expressed as percentage of dry biomass and concentration of lipids per milliliter of culture medium were analyzed by one-way ANOVA, and mean separations were performed by LSD tests $(\mathrm{P}<0.05)$. The strain which accumulated significantly higher amounts of intracellular lipids was selected. For the selected strain, biomass (as dry weight), ammonia, nitrate and glycerol concentration were determined at different times after inoculation ( $0,20,44,67,90,114$ and 138 hours). Intracellular saponifiable lipids were evaluated daily from the second to the sixth day. Ammonia was determined using the commercial kit UREA/BUN-COLOR (Biosystems COD 11536) and nitrate was analyzed by HPLC with a IC-Pack Anion column (Waters) maintained at $40{ }^{\circ} \mathrm{C}$, using phosphate buffer $(0,01 \mathrm{M}, \mathrm{pH} 6,8)$ as mobile phase at a flux of $1,2 \mathrm{~mL}$ $\min ^{-1}$ and a UV detector (Shimadzu SPD-10AV UV-VIS Detector) at $210 \mathrm{~nm}$. Glycerol was determined by HPLC using an Aminex HPX-87C (Bio-Rad), 250 x 4 mm column, at $67{ }^{\circ} \mathrm{C}$ with water as mobile phase and a RID-10A Shimadzu detector.

\subsection{Growth of the Selected Strain in the Presence of Crude Glycerol}

Growth in the presence of pure and crude glycerol was compared for the selected strain. Lipid accumulation at $5^{\text {th }}$ day was also determined in each case. Medium composition and growth conditions were the same used in the previous experiments. Crude glycerol (purity 80\%) containing methanol $(3 \%)$; water $(3 \%)$ and organic matter not glycerol (13\%) as impurities, was provided by ALUR, a Uruguayan biodiesel producing industry. The culture medium was prepared to reach a glycerol concentration of $40 \mathrm{gl}^{-1}$. Since turbidity of the medium containing crude glycerol was high, in this case, growth was evaluated by direct microscopic counting of yeast cells in a Neubauer chamber at different times $(0,12,22,46$, and 67 hours). Growth was expressed as the $\ln (\mathrm{Xi} / \mathrm{X} 0)$ being $\mathrm{Xi}$ the yeast concentration at each time and $\mathrm{X} 0$ the initial concentration. The experiment was repeated three times. Data of yeast growth in pure and crude glycerol at each time point were compared by one-way ANOVA, and mean separations were performed by LSD tests. Differences at $\mathrm{P}<0.05$ were considered significant.

\subsection{Effect of Crude Glycerol Concentrations on Yeast Growth and Lipid Accumulation}

The selected strain was grown in presence of different concentrations of crude glycerol representing 40, 60, 80 and $100 \mathrm{~g} \mathrm{l}^{-1}$ of glycerol in the culture medium, in the same growth conditions ase described above. Biomass, as dry weight, and the content of intracellular saponifiable lipids at the fifth day was determined. The experiment was repeated twice. Biomass and lipid data were analyzed by one-way ANOVA, and mean separations were performed by LSD tests $(\mathrm{P}<0.05)$

\section{Results}

\subsection{Isolation and Screening for Oleaginous Yeasts}

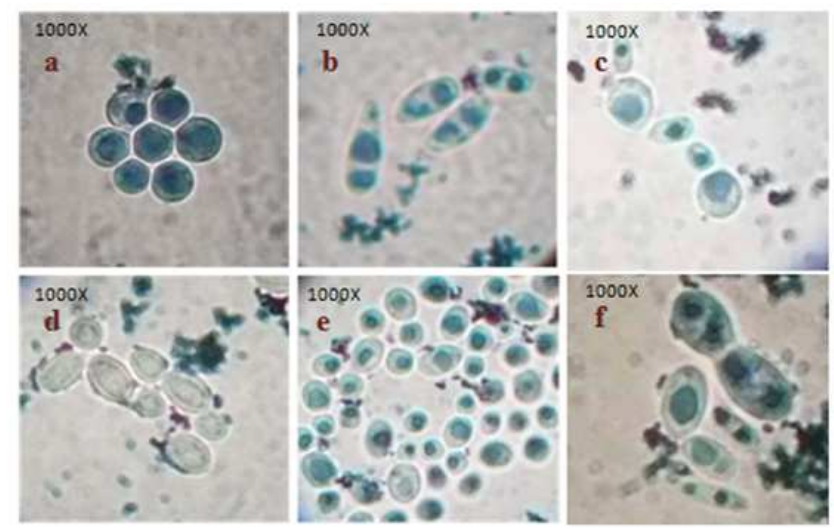

Fig. 1. Optical microscopy photographs of yeast isolates stained with Sudan Black B. Lipid globules stained in bluish-gray are observed in the oleaginous yeast $(a),(b),(c),(e)$ and $(f)$. Saccharomyces cerevisiae $(d)$ previously identified as not oleaginous yeast was used as a negative control. a) Strain S31; b) Strain AT17; c) Strain S12R; d) S. cerevisiae strain; e) Strain AT7; f) Strain PL1

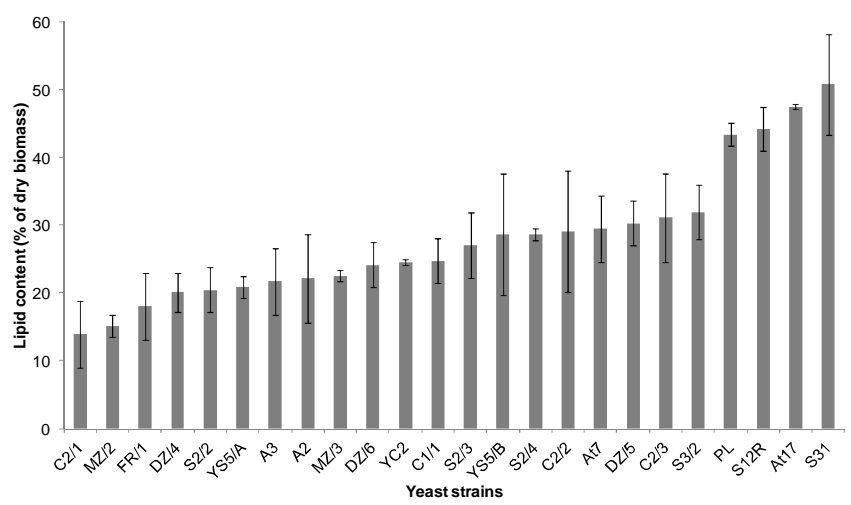

Fig. 2. Lipid content of 24 potential oleaginous yeast strains, after a 6-day culture at $150 \mathrm{rpm}$ and $28^{\circ} \mathrm{C}$. Data are mean \pm standard deviation (error bars) of two independent experiments $(\alpha=0.05)$

Thirteen isolates were recovered from soil samples and twelve were obtained from fruit surfaces. In total, thirty yeast isolates (including the strains from our culture collection) were screened to determine their capacity to accumulate intracellular lipids by the Sudan Black B staining method. Twenty-four isolates were identified as potential lipid producers while six isolates, which did not show stained fat droplets inside the cells, were classified as non oleaginous and discarded. Figure 1 shows a microscopic view (10X100) of smears of four different strains stained with Sudan Black B. In three of them, stained internal oil droplets can be observed. Intracellular content of saponifiable lipids was 
determined for the potential oleaginous isolates (Fig. 2). As a result, the isolates PL, S12R, At17 and S31 were selected since they showed significantly higher lipid content (43.2, 44.1, 47.1 and 50.5\%; $\mathrm{p}<0.0001)$.

\subsection{Determination of Fatty Acid Profiles}

The fatty acids profiles of lipids extracted from the four selected strains are shown in Table 1. Similar profiles were obtained for the four strains. Saturated acids such as myristic (14:0), palmitic (16:0) and stearic (18:0) were found in lipids from all strains. Behenic acid (22:0) was produced only by At17 and PL1 strains. The unsaturated acids were represented by palmitoleic (16:1), oleic (18:1), linoleic (18:2) and linolenic (18:3) acids. In all cases, palmitic and oleic acids were the two major components. Moreover, linolenic acid was found in low concentrations (less than 3\%), fatty acids with more than four double bounds were absent and calculated cetane numbers were above 45 in all cases (Table 1). Palmitoleic $\left(\mathrm{M}^{+} 268\right)$ and behenic $\left(\mathrm{M}^{+} 354\right)$ acids were identified by GC-MS.

Table 1. Fatty acid composition (\%) and cetane number of 4 selected oleaginous yeast strains after a 5-day culture using glucose as carbon source.

\begin{tabular}{llllllllll}
\hline Strain & C14:0 & C16:0 & C16:1 & C18:0 & C18:1 & C18:2 & C18:3 & C22:0 & CN \\
\hline At17 & 0.91 & 20.35 & 0.33 & 7.24 & 56.31 & 11.81 & 2.15 & 0.90 & 55.6 \\
PL & 0.79 & 19.39 & 0.22 & 6.22 & 60.37 & 10.76 & 1.53 & 0.72 & 55.6 \\
S12R & 1.50 & 17.00 & 5.80 & ND & 64.70 & 8.90 & 2.10 & ND & 53.7 \\
S31 & 0.23 & 27.29 & 0.96 & 9.08 & 53.91 & 8.52 & ND & ND & 58.4 \\
\hline
\end{tabular}

CN: Cetane number ND: Not detected.

\subsection{Identification and Characterization of the Selected Oleaginous Yeast Strains}

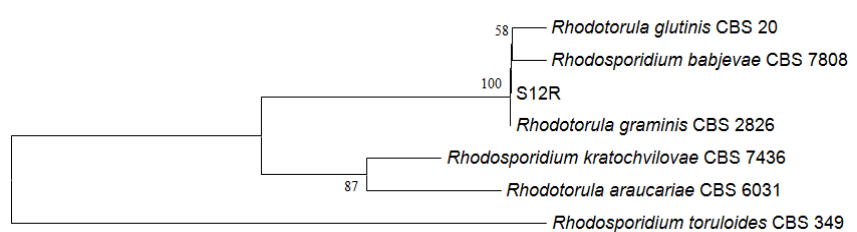

Fig. 3. Phylogenetic tree of the D1/D2 region sequences of strain $S 12 R$ and related species. The tree was constructed using the Neighbor-Joining method. Bootstrap values (1000 tree interactions) are indicated at the nodes

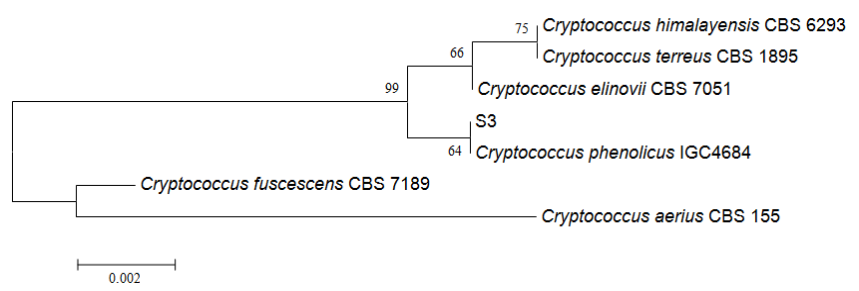

Fig. 4. Phylogenetic tree of the D1/D2 region sequences of S31 strain and related species. The tree was constructed using the Neighbor-Joining method. Bootstrap values (1000 tree interactions) are indicated at the nodes

Molecular analysis of the nucleotide sequences of D1/D2 and ITS 1-5.8S-ITS2 regions was performed for the identification of the strains selected. Both sequences from S12R strain showed a $100 \%$ homology with the same sequences of Rhodotorula graminis CBS 2826 (type strain). Relatedness of sequences of D1/D2 region obtained from the isolate $\mathrm{S} 12 \mathrm{R}$ to those corresponding to other closely related species retrieved from GenBank is presented in the phylogenetic tree shown in Fig. 3. The tree obtained by analyzing ITS1-ITS2 domain is not shown since the same relationships among species were assessed. According to the obtained results strain $\mathrm{S} 12 \mathrm{R}$ was identified as $R$. graminis.
The nucleotide sequences corresponding to the D1/D2 and the ITS1-ITS2 domains of rDNA of S12R have been deposited in GenBank under the following accession numbers: KM207221 and KM207222 respectively. For S31 strain, the molecular analysis showed that the amplified sequences were identical to those corresponding to Cryptococcus phenolicus (IGC4684) (type strain), Relatedness of sequences D1/D2 region obtained from the isolate S31 to those corresponding to other closely related species retrieved from GenBank is presented in the phylogenetic tree shown in Fig. 4. Only phylogenetic tree derived from analysis of 26S rDNA domain D1/D2 is shown as the tree obtained by analyzing ITS1-ITS2 domain showed essentially the same relationships among isolates and species. The nucleotide sequences corresponding to the D1/D2 and the ITS1-ITS2 domains of rDNA of S31 have been deposited in GenBank under the following accession numbers: KM213395 and KM213396 respectively. The other two selected strains (At17 and PL1) were identified in previous studies[5], as Leucosporidium scottii and Cystofilobasidium infirmominiatum, respectively.

\subsection{Intracellular Lipid Accumulation in Presence of Glycerol}

Strains At17, PL1 and S12R identified as L. scottii, C. infirmonimiatum and $R$. graminis were able to grow in presence of glycerol as the only carbon source. Fatty acid profiles obtained for each strain when grown in presence of glucose or glycerol were very similar, resulting in similar cetane numbers. However, in case of At17 and PL1 strains, arachidic acid (20:0) was produced in presence of glycerol, but not in presence of glucose (Table 2). The percentage of internal lipids per gram of biomass, in both substrates was significantly higher in the case of strains PL1 and S12R, with no differences between them. However, when analyzing the 
concentration of microbial lipids obtained per liter of culture medium, the highest value corresponded to the strain S12R. According to this result $R$. graminis $\mathrm{S} 12 \mathrm{R}$ was selected for further studies. Figure 5 shows the time course of a batch culture of the selected yeast growing in presence of glycerol as carbon and energy source. Lipid production was assessed after nitrogen depletion when glycerol still remained in the culture medium. Biomass and lipid concentration was not significantly different at time $114 \mathrm{~h}$ and $138 \mathrm{~h}$, so in the conditions used in this work, a five days culture was appropriate to get the maximum concentration of lipids $(5.0 \mathrm{~g}$ $1^{-1}$ ) which implies a lipid yield of $0.13 \mathrm{~g}$ of lipids per $\mathrm{g}$ of glycerol consumed.

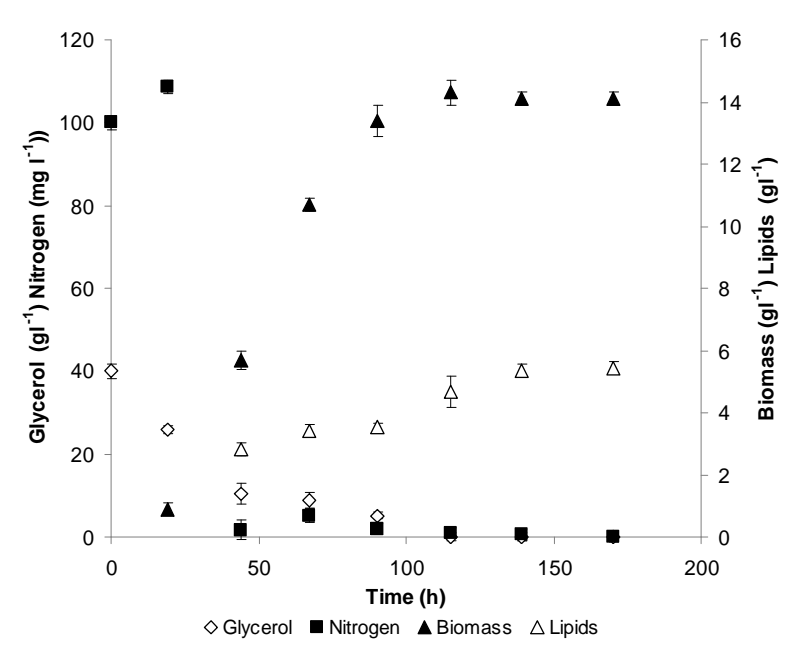

Fig. 5. Time course of lipid concentration (open triangle), biomass concentration (filled triangle), nitrogen (filled square) and glycerol (open diamond) consumption of $R$. graminis $S 12 R$ in presence of glycerol as carbon and energy source. Data are mean \pm standard deviation (error bars) of three assays

Table 2. Lipid content ( $\mathrm{g} / \mathrm{l} ; \%$ according to total cell dry weight), fatty acid composition (\%) and cetane number of selected oleaginous yeast strains after a 5 day culture using glycerol as carbon source.

\begin{tabular}{|c|c|c|c|c|c|c|c|c|c|c|c|c|}
\hline Strain & Lipids $(\%)^{*}$ & Lipids $\left(\mathrm{gl}^{-1}\right)^{*}$ & C14:0 & C16:0 & C16:1 & C18:0 & C18:1 & C18:2 & C18:3 & C20:0 & C22:0 & $\mathrm{CN}$ \\
\hline At17 & $32 \pm 2^{\mathrm{a}}$ & $3.8 \pm 0.2^{\mathrm{B}}$ & 0.95 & 20.5 & 0.35 & 13.28 & 45.88 & 13.70 & 2.76 & 0.55 & 0.97 & 56.8 \\
\hline PL1 & $39 \pm 1^{b}$ & $3.0 \pm 0.2^{\mathrm{A}}$ & 0.92 & 19.4 & 0.41 & 11.76 & 46.82 & 14.73 & 3.30 & 0.50 & 1.02 & 55.9 \\
\hline S12R & $41.4 \pm 0.2^{b}$ & $5.0 \pm 0.0^{\mathrm{C}}$ & 1.32 & 16.9 & 4.25 & 2.36 & 50.16 & 13.00 & 5.00 & ND & 0.48 & 55.5 \\
\hline
\end{tabular}

$\mathrm{CN}$ : Cetane number. ND: Not detected. *Data are mean \pm standard deviation of two assays.

a,b,c A,B,C Values followed by the same letter do not differ statistically $(\mathrm{P} \leq 0.05)$.

\subsection{Growth and Lipid Accumulation in Presence of Pure and Crude Glycerol}

Growth and lipid accumulation in the presence of pure and crude glycerol was compared for the selected strain. Growth profiles were very similar (Table 3 ). There were no significant differences in yeast growth obtained in both cases throughout the experiment. On day 5 intracellular lipid concentration in yeasts grown in the presence of crude glycerol was $5.3 \mathrm{~g} \mathrm{l}^{-1}$, which was not significantly different from the lipid content obtained in the presence of pure glycerol $\left(5.0 \mathrm{~g} \mathrm{l}^{-1}\right)$.

Table 3. Growth of $R$. graminis $S 12 R$ in the presence of pure and crude glycerol expressed as the $\ln \left(X_{i} / X_{0}\right)$ being $X_{i}$ the yeast concentration at each time and $X_{0}$ the initial concentration

\begin{tabular}{lll}
\hline \multirow{2}{*}{ Time (h) } & $\ln \left(\mathbf{X}_{\mathbf{i}} / \mathbf{X}_{\mathbf{0}}\right)$ & \\
\cline { 2 - 3 } & Pure glycerol & Crude gycerol \\
\hline 12 & $2,2 \pm 0.3^{\mathrm{a}}$ & $2,8 \pm 0.3^{\mathrm{a}}$ \\
22 & $2,6 \pm 0.6^{\mathrm{a}}$ & $3,2 \pm 0.2^{\mathrm{a}}$ \\
46 & $3,4 \pm 0.6^{\mathrm{a}}$ & $3,9 \pm 0.3^{\mathrm{a}}$ \\
67 & $3,7 \pm 0.6^{\mathrm{a}}$ & $3,9 \pm 0.2^{\mathrm{a}}$ \\
120 & $3,7 \pm 0.6^{\mathrm{a}}$ & $3,9 \pm 0.3^{\mathrm{a}}$ \\
\hline
\end{tabular}

Values followed by the same letter are not statistically different $(\mathrm{P} \leq 0.05)$

\subsection{Effect of Crude Glycerol Concentrations on Yeast Growth}

Biomass production at 40 or $60 \mathrm{~g} \mathrm{l}^{-1}$ of crude glycerol was not significantly different. However, higher concentrations resulted in an inhibition of yeast growth (Table 4). Intracellular lipid concentration was determined in cultures obtained at 40 or $60 \mathrm{~g} \mathrm{l}^{-1}$ which resulted significantly different, being higher $\left(5.9 \mathrm{gl}^{-1}\right)$ when a higher concentration of glycerol was used (Table 4).

Table 4. Biomass and lipids production of $R$. graminis $S 12 R$ in presence of different concentrations of crude glycerol after a 5-day culture.

\begin{tabular}{lll}
\hline Glycerol concentration $\left(\mathbf{g l}^{-\mathbf{1}}\right)$ & Biomass $\left(\mathbf{g l}^{\mathbf{1}}\right)$ & Lipids $\left(\mathbf{g l}^{\mathbf{- 1}}\right)$ \\
\hline 40 & $12.5 \pm 0.4^{\mathrm{a}}$ & $5.4 \pm 0.1^{\mathrm{a}}$ \\
60 & $13.6 \pm 0.4^{\mathrm{a}}$ & $5.9 \pm 0.0^{\mathrm{b}}$ \\
80 & $11.1 \pm 0.0^{\mathrm{b}}$ & nd \\
100 & $10.6 \pm 0.4^{\mathrm{b}}$ & nd \\
\hline
\end{tabular}

The experiment was repeated twice. Biomass and Lipids data were analyzed by one-way ANOVA, and mean separations were performed by LSD tests. ${ }^{\mathrm{a}, \mathrm{b}}$ Differences at $\mathrm{P}<0.05$ were considered significant. nd, not determined.

\section{Discussion}

In the present study a screening of oleaginous yeasts from different habitats nearby Montevideo, Uruguay, was carried 
out. More than $75 \%$ of the obtained isolates were characterized as oleaginous according to Li el al. [4], since they were capable of yielding lipid content higher than $20 \%$ on their dry weight, These results confirm the wide distribution of these microorganisms as stated by Rossi et al. [19]. Moreover, two of the psycrotrophic isolates from Antarctica resulted oleaginous. This finding was not surprising since the presence of oleaginous yeasts and molds in extreme cold environments like the Tibetian plateau has already been reported [20]. Although many of the isolates were characterized as oleaginous, only four isolates were able to accumulate a significantly higher amount of intracellular lipids, with levels exceeding the $40 \%$ of cell dry weight. One of them, was identified as Rhodotorula graminis which has been already recognized as an oleaginous species [21]. However, the other three isolates belong to species, which have not been reported as oleaginous before. In fact, this work constitutes the first report of Cryptococcus phenolicus, Cystofilobasidium infirmominiatum and Leucosporidium scottii as oleaginous species.

Biodiesel must meet certain requirements to be used as a fuel, some of which rely on the fatty acid mixture used in biodiesel production. In Uruguay, standards for biodiesel indicate that cetane number must be higher than 45 , and no more than $12 \%$ of linolenic acid esters and $1 \%$ of esters from polyunsaturated acids (more than 4 double bonds) is admitted [22]. The fatty acid profiles obtained from the yeast selected in this paper met Uruguayan standards for biodiesel when grown with different carbon sources, including crude glycerol. Thus, the use of that secondary product obtained from biodiesel production appears as an option of carbon source to produce oleaginous yeasts, in order to reduce the costs. It was demonstrated that a concentration of the substrate to achieve $60 \mathrm{~g} \mathrm{l}^{-1}$ of glycerol could be incorporated in the culture medium, without affecting yeast growth. Moreover, a higher concentration of intracellular lipids was achieved in such conditions, which was probably due to a higher $\mathrm{C} / \mathrm{N}$ ratio in the culture medium. Last year, in Uruguay, 50000 ton of biodiesel were produced by ALUR (National Supply Company) to be incorporated in a proportion of $7 \%$ to diesel for locomotive use[23]. However, the production cost of biodiesel is not economically competitive with petroleum-based fuel because of the relatively high cost of the oil feedstock. Moreover, the use of vegetable oils as raw material for biodiesel production competes with edible oils, thus leading to a soaring of food price. According to Duarte [24] about $600 \mathrm{~kg}$ of oil can be produced annually from 1 hectare of soybean, which can be used for biodiesel production. Since a lipid production of 5 $\mathrm{gl}^{-1}$ was obtained from $R$. graminis $\mathrm{S} 12 \mathrm{R}$ growing in crude glycerol, about 120000 liters of such culture would be required for the production an amount of oil equivalent to that obtained from 1 hectare of soybean. Even if 120000 liters seems a very high volume, it should be considered that yeast culture for oil production takes only 5 days, while soybean crop is annual. Lipid yield on glycerol obtained in the present study showed similar results when compared with other studies[25, 26]. However, the lipid production could be improved. In this regard, Lin et al. [27] designed a two-stage fermentation process to obtain a lipid concentration $67.9 \mathrm{gl}^{-1}$ from the yeast Lipomyces starkeyi. The optimization of the growing conditions for $R$. graminis $\mathrm{S} 12 \mathrm{R}$ will be the next step in order to obtain the maximum lipid yield.

\section{Acknowledgements}

The authors would like to extend their thanks to the Agencia Nacional de Investigación e Innovación (ANII) and the Organization for the Prohibition of Chemical Weapons (OPCW) for funding this work.

\section{References}

[1] Fortenbery, T. R.: Biodiesel Feasibility Study: An Evaluation 468 of Biodiesel Feasibility in Wisconsin, University of Wisconsin-Madison, Department of Agricultural \& Applied Economics, Staff Paper, 481 (2005).

[2] Yazdani, S. S. and Gonzalez, R.: Anaerobic fermentation of glycerol: a path to economic viability for the biofuels industry, Current Opinion in Biotechnology, 18, 213-219 (2007).

[3] Chi, Z., Pyle, D., Wen, Z., Frear, C. and Chen, S.: A laboratory study of producing docosahexaenoic acid from biodieselwaste glycerol by microalgal fermentation, Process Biochemistry, 42, 1537-1545 (2007).

[4] Li, Y., Zhao, Z. and Bai, F.: High-density cultivation of oleaginous yeast Rhodosporidium toruloides Y4 in fed-batch culture, Enzyme and Microbial Technology, 41, 312-317 (2007).

[5] Vero, S., Garmendia, G., González, M. B., Bentancur, O. and Wisniewski, M.: Evaluation of yeasts obtained from Antarctic soil samples as biocontrol agents for the management of postharvest diseases of apple (Malus $\times$ domestica), FEMS Yeast Research, 13, 189-199 (2013).

[6] Thakur, M. S., Prapulla, S. G. and Karanth, N. G.: Microscopic observation of Sudan Black B staining to monitor lipid production by microbes, Journal of Chemical Technology and Biotechnology, 42, 129-134 (1988).

[7] Thakur, M. S., Prapulla, S. G. and Karanth, N. G.: Estimation of intracellular lipids by the measurement of absorbance of yeast cells stained with Sudan Black B, Enzyme and Microbial Technology, 11, 252-254 (1989).

[8] Vorapreeda, T., Thammarongtham, C., Cheevadhanarak, S. and Laoteng, K.: Alternative routes of acetyl-CoA synthesis identified by comparative genomic analysis: Involvement in the lipid production of oleaginous yeast and fungi, Microbiology, 158, 217-228 (2012).

[9] Burja, A. M., Armenta, R. E., Radianingtyas, H. and Barrow, C. J.: Evaluation of fatty acid extraction methods for Thraustochytrium sp. ONC-T18, Journal of Agricultural and Food Chemistry, 55, 4795-4801 (2007).

[10] Azam, M. M., Waris, A. and Nahar, N. M.: Prospects and potential of fatty acid methyl esters of some non-traditional seed oils for use as biodiesel in India, Biomass and Bioenergy, 29, 293-302 (2005). 
[11] Schena, L., Ippolito, A., Zahavi, T., Cohen, L., Nigro, F. and Droby, S.: Genetic diversity and biocontrol activity of Aureobasidium pullulans isolates against postharvest rots, Postharvest Biology and Technology, 17, 189-199 (1999).

[12] White, T. J., Bruns, T., Lee, S. and Taylor, J.: Amplification and direct sequencing of fungal ribosomal RNA genes for phylogenetics, PCR protocols: a guide tomethods and applications Innis M, Gelfand D, Sninsky J, White T (eds) Academic Press,New York, Chapter 38, 315-322 (1990).

[13] Peterson, S. W. and Horn, B. W.: Penicillium parvulum and Penicillium georgiense, sp. nov., isolated from the conidial heads of Aspergillus species, Mycologia, 101, 71-83 (2009).

[14] Tamura, K., Peterson, D., Peterson, N., Stecher, G., Nei, M. and Kumar, S.: MEGA5: Molecular evolutionary genetics analysis using maximum likelihood, evolutionary distance, and maximum parsimony methods, Molecular biology and evolution, 28, 2731-2739 (2011).

[15] Jukes, T. and Cantor, C.: Evolution of protein molecules, H. M. Munro, ed. Mammalian Protein Metabolism. Academic Press, New York, NY., 21-132 (1969).

[16] Saitou, N. and Nei, M.: The neighbor-joining method: a new method for reconstructing phylogenetic trees, Molecular biology and evolution, 4, 406-425 (1987).

[17] Felsenstein, J.: Phylogenies and the comparative method, American Naturalist, 125, 1-15 (1985).

[18] Kurtzman, C. P. and Fell, J. W.: The Yeasts: A Taxonomic Study, Kurtzman CP, JW Fell (Eds), Elsevier, Amsterdam, 4th edition, 1-1055 (1998).

[19] Rossi, M., Amaretti, A., Raimondi, S. and Leonardi, A.: Getting Lipids for Biodiesel Production from Oleaginous Fungi, Biodiesel - Feedstocks and Processing Technologies, Dr. Margarita Stoytcheva (Ed.), Chapter 4, (2011).
[20] Li, S. L., Lin, Q., Li, X. R., Xu, H., Yang, Y. X., Qiao, D. R. and Cao, Y.: Biodiversity of the oleaginous microorganisms in Tibetan Plateau, Brazilian Journal of Microbiology, 43, 627634 (2012).

[21] Galafassi, S., Cucchetti, D., Pizza, F., Franzosi, G., Bianchi, D. and Compagno, C.: Lipid production for second generation biodiesel by the oleaginous yeast Rhodotorula graminis, Bioresource Technology, 111, 398-403 (2012).

[22] UNIT1100:2009: Biodiesel (B100) Combustible para uso puro o en mezcla con destilados medios de petróleo- Requisitos (2009).

[23] ALUR: Actualización Biodiesel. http://www.alur.com.uy/biodiesel-actualizacion.html. http://www.alur.com.uy/biodiesel-actualizacion.html (2014).

[24] Duarte, S. H., de Andrade, C. C. P., Ghiselli, G. and Maugeri, F.: Exploration of Brazilian biodiversity and selection of a new oleaginous yeast strain cultivated in raw glycerol, Bioresource Technology, 138, 377-381 (2013).

[25] Papanikolaou, S., Fakas, S., Fick, M., Chevalot, I., GaliotouPanayotou, M., Komaitis, M., Marc, I. and Aggelis, G.: Biotechnological valorisation of raw glycerol discharged after bio-diesel (fatty acid methyl esters) manufacturing process: Production of 1,3-propanediol, citric acid and single cell oil, Biomass and Bioenergy, 32, 60-71 (2008).

[26] Fakas, S., Papanikolaou, S., Batsos, A., Galiotou-Panayotou, M., Mallouchos, A. and Aggelis, G.: Evaluating renewable carbon sources as substrates for single cell oil production by Cunninghamella echinulata and Mortierella isabellina, Biomass and Bioenergy, 33, 573-580 (2009).

[27] Lin, J., Shen, H., Tan, H., Zhao, X., Wu, S., Hu, C. and Zhao, Z. K.: Lipid production by Lipomyces starkeyi cells in glucose solution without auxiliary nutrients, Journal of Biotechnology, $152,184-188$ (2011). 